\section{Case Reports in Dermatology}

This article is licensed under the Creative Commons Attribution-NonCommercial 4.0 International License (CC BY-NC) (http://www.karger.com/Services/OpenAccessLicense) Usage and distribution for commercial purposes requires written permission.

\title{
Adenolipoma of the Skin: A Report of 11 Cases
}

\author{
Rawan Amir ${ }^{a}$ Salwa Sheikh ${ }^{b}$ \\ almam Abdulrahman Bin Faisal University, Dhahran, Saudi Arabia; bPathology Services \\ Division, Johns Hopkins Aramco Healthcare, Dhahran, Saudi Arabia
}

\section{Keywords}

Adenolipoma $\cdot$ Lipoma $\cdot$ Neoplasm

\begin{abstract}
Introduction: Adenolipoma is a relatively recently described lesion representing a rare variant of lipoma in which eccrine glands and ducts are found interspersed amongst mature adipose tissue. This benign lesion was first described by Hitchcock et al. in 1993 [J Am Acad Dermatol 1993;29:82-85]. The next case series was written by Ait-Ourhrouil and Grosshans [Ann Dermatol Venerol 1997;124:845-848] in which they deemed adenolipoma a misnomer based on the pathophysiology of the lesion and suggested the name peri-sudoral lipoma instead. According to our knowledge, this case series would be the third in the literature to report cases of adenolipoma in an attempt to increase awareness of this entity. Methods: We performed a retrospective review of adenolipoma cases from 2004 to 2014 at our institute. Results and Conclusions: Upon review of all adenolipomas diagnosed at our institute between 2004 and 2014, 11 cases in total were identified. Histologically, these lesions consisted of an admixture of adult-like adipose tissue and scattered eccrine glands and ducts. 4 cases also showed areas of myxoid changes; 2 showed scattered mast cells amongst the adipose tissue, and only 1 showed the presence of apocrine glands in addition to the eccrine glands and ducts. The female to male ratio was 6:5. The typical age ranged from 41 to 53 years.
\end{abstract}


Adenolipomas were seen arising from various locations including the thighs, gluteal region, lower leg, shoulder, chest, and trunk. The majority of cases were not fully encapsulated and the size varied from 0.7 to $5.8 \mathrm{~cm}$.

(C) 2018 The Author(s)

Published by S. Karger AG, Basel

\section{Introduction}

Adenolipoma is a rare variant of lipoma which contains eccrine glands and ducts amongst mature adipose tissue. It is a benign lesion similar to lipoma and was initially described in 1993 by Hitchcock et al. [1] in a case series. This was followed by another case series by Ait-Ourhrouil and Grosshans [2] in which they disagreed with the name given to this lesion and recommended calling it peri-sudoral lipoma instead. Clinically, adenolipomas present in a similar manner to lipomas. However, the presence of eccrine glands within the adipose tissue helps to histologically distinguish adenolipomas from common lipomas. Awareness of this entity is important so that it can be identified as a variant of lipoma by pathologists and as a benign lesion by clinicians. The recommended treatment, surgical excision, and prognosis are like that of typical lipomas. According to our knowledge, this case series is the third in the literature describing and reporting this entity.

\section{Materials and Methods}

A retrospective study of all cases of adenolipoma at our institute from 2004 to 2014 was performed. A total of 11 cases were identified (Table 1).

\section{Results}

11 cases of adenolipoma were identified at our institute between 2004 and 2014. Histologically, these lesions consisted of lobules of mature adipose tissue with scattered eccrine glands and ducts. 4 cases had focal myxoid changes (36\%); 2 cases showed scattered mast cells amongst the adipose tissue (18\%), and only 1 case had apocrine glands present in addition to the eccrine glands and ducts (9\%). 6 cases were found in females (54.5\%), while the remaining 5 were in male patients (45.5\%). Most cases $(82 \%)$ were diagnosed in patients between 41 and 53 years of age, while the remaining 2 cases $(18 \%)$ were diagnosed in an 18-year-old and a 79-year-old. The location of the lesions varied; 3 were located in the left thigh $(27 \%), 2$ in the right thigh (18\%), 2 in the right gluteal region $(18 \%), 1$ in the right lower leg (9\%), 1 in the left shoulder (9\%), 1 in the left chest (9\%), and 1 in the left trunk $(9 \%)$. The majority of the lesions were not fully encapsulated. The size of the lesions varied from 0.7 to $5.8 \mathrm{~cm}$. Immunohistochemistry was performed on 1 case and showed cytokeratin highlighting the eccrine ducts which were deeply embedded into the fat. 


\section{Discussion}

Adenolipoma of the skin is a recently discovered benign entity first described in 1993 by Hitchcock et al. [1]. It is a variant of lipoma which consists of an admixture of adipose tissue and eccrine sweat glands, hence the name adenolipoma. However, in 1997, the name perisudoral lipoma was suggested by Ait-Ourhrouil and Grosshans [2] since they believed that adenolipoma is a misnomer due to the fact that this lesion originates from adipose tissue around the eccrine glands without proliferation of the glands or ducts themselves. Adenolipomas are typically found in patients aged 25-75 years, with the average being 50 years of age [3].

Clinically, adenolipomas present as a single, slow-growing, painless lump. They are very similar in presentation to typical lipomas. Adenolipomas are most commonly found arising from the thigh, followed by the arms, shoulders, and chest [4]. The size typically ranges from 1 to $6 \mathrm{~cm}$.

By gross examination, adenolipomas appear to be soft, lobulated, yellow masses. Histologically, they consist of lobules of adipose tissue that are larger than those of typical subcutaneous tissue. Dispersed amongst these adipocyte clusters are adult-like eccrine glands and ducts (Fig. 1, 2). These eccrine glands and ducts may show epithelial hyperplasia, cystic duct dilatation, and squamous or clear cell metaplasia [5]. Rarely, apocrine glands may also be found as noted in one of our cases [6]. The first case of apocrine glands visualized within an adenolipoma was reported in 2005 by Antúnez et al. [7] in an adenolipoma of the elbow. Most reported cases of adenolipomas have an encircling capsule; however, only 1 of the 11 cases in our case study was encapsulated. They typically arise from the dermis or subcutaneous fat. As for adenolipomas which involve the dermis, they are found at a deeper level than the normal eccrine glands. The distance between the dermis and eccrine sweat glands in both patients and adenolipoma-free people was measured by Ait-Ourhrouil and Grosshans [2]. They concluded that the distance is much smaller in normal skin than in adenolipomas.

Histological features in correlation with clinical presentation are the mainstay for the diagnosis of adenolipoma with adipose cells mixed with eccrine glands and ducts. Ancillary studies such as special stains and immunohistochemistry may help highlight features but are not required to make a diagnosis. Eccrine glands are composed of secretory epithelial cells surrounded by myoepithelial cells. The secretory cells contain intracytoplasmic granules and intraluminal secretions which stain periodic acid-Schiff positive and are positive for epithelial markers such as cytokeratin. The surrounding myoepithelial cells are actin, S-100, and p63 positive [5].

The most common differential diagnoses of adenolipoma include the typical lipoma, nevus lipomatosus superficialis, eccrine angiomatous hamartoma, spindle cell lipoma, and cutaneous myxolipoma. Adenolipomas and lipomas have a very similar presentation; they both present in around the same age range and tend to spare the more distal regions of the body. However, adenolipomas are typically smaller in size. Occasionally, a common subcutaneous lipoma may compress the dermis giving an appearance very similar to that of an adenolipoma. Yet the presence of eccrine glands around the lesion as opposed to being found within the lesion hints towards a diagnosis of a common lipoma. 
Nevus lipomatosus superficialis presents in quite a different manner from adenolipomas. As opposed to being a single lesion it usually presents as multiple papules or nodules with a wrinkled or smooth surface arranged in a linear fashion commonly on the hip or buttock. It presents at birth or during infancy. Rarely, it may present as a solitary mass but they are more likely to be diagnosed as polypoid fibrolipomas. Histologically, collections of adipocytes amidst collagen bundles are found in the dermis. The rest of the dermis appears to be completely normal; however, the number of fibroblasts, the density of the bundles of collagen, and the vascularity are higher than those of normal skin. An abundance of mast cells and perivascular inflammation are also noted.

Eccrine angiomatous hamartomas usually present as a solitary lesion on the lower limbs at birth. On microscopy, the deeper layers of the dermis show a raised number of eccrine structures surrounded or intermingled with capillary channels. Some fatty tissue may also be seen in the rare lipomatous variant [3].

Spindle cell lipomas are benign lesions typically affecting the posterior neck, upper back, and shoulders. Microscopy shows three main constituents: adipose cells, spindle cells, and collagen. Occasionally, myxoid matrix may be seen, while with cutaneous myxolipoma typical microscopy shows adipocytes with well-developed myxoid areas.

The reasons behind easy misdiagnosis of adenolipomas are quite simple. Fragmented specimens may cause a problem as it is not always possible to properly assess the location of the eccrine glands. Also, the presence of eccrine glands in the periphery of the lesion makes it difficult to distinguish glands actually involved in the tumor from normal glands lying adjacent to the tumor. These diagnostic pitfalls can be avoided by serial sectioning of the lesion to confirm the presence of eccrine glands and/or ducts within the lesion. In addition, awareness of the pathologist of this variant is significant in order to be able to recognize it as such.

The treatment and prognosis of adenolipomas are very similar to typical lipomas. Surgical excision is the recommended treatment of choice, and recurrence is rare.

\section{Conclusion}

A rare variant of lipomas which contain eccrine glands and ducts embedded within mature adipose tissue is termed adenolipoma. This entity clinically presents as a lump that looks like a lipoma. Histologically, it can be distinguished from the common lipoma by the presence of eccrine glands and ducts. Adenolipomas typically occur in people between 25 and 75 years of age. Increased awareness of this lesion is important for pathologists in order to be able to identify it and for clinicians to recognize it as a benign neoplasm. The management and prognosis are both very similar to that of common lipomas.

\section{Disclosure Statement}

There is no conflict of interest or financial acknowledgments for this case series. 


\section{References}

1 Hitchcock MG, Hurt MA, Santa Cruz DJ. Adenolipoma of the skin: a report of nine cases. J Am Acad Dermatol. 1993 Jul;29(1):82-5.

2 Ait-Ourhrouil M, Grosshans E. [Peri-sudoral lipoma]. Ann Dermatol Venereol. French. 1997;124(12):845-8.

3 Del Agua C, Felipo F. Adenolipoma of the skin. Dermatol Online J. 2004 Oct;10(2):9.

4 Karoui S, Badri T, Benmously R, Ben Brahim E, Chadli-Debbiche A, Mokhtar I et al. Adenolipoma of the skin. Pathologica. 2011 Dec;103(6):343-5.

5 Lee HS, Song YS. Adenolipoma of the skin arising at neck region. Korean J Pathol. 2012 Dec;46(6):587-9.

6 Kazakov DV, Spagnolo DV, Kacerovska D, Kempf W, Michal M. Cutaneous adenolipoma: extending the spectrum of changes in the lipomatous and epithelial components. Am J Dermatopathol. 2011 Feb;33(1):569.

7 Antúnez P, Santos-Briz A, Muñoz E, Fernández M, de Unamuno P, Bullón A. Cutaneous apocrine cystic adenolipoma. Am J Dermatopathol. 2005 Jun;27(3):240-2.

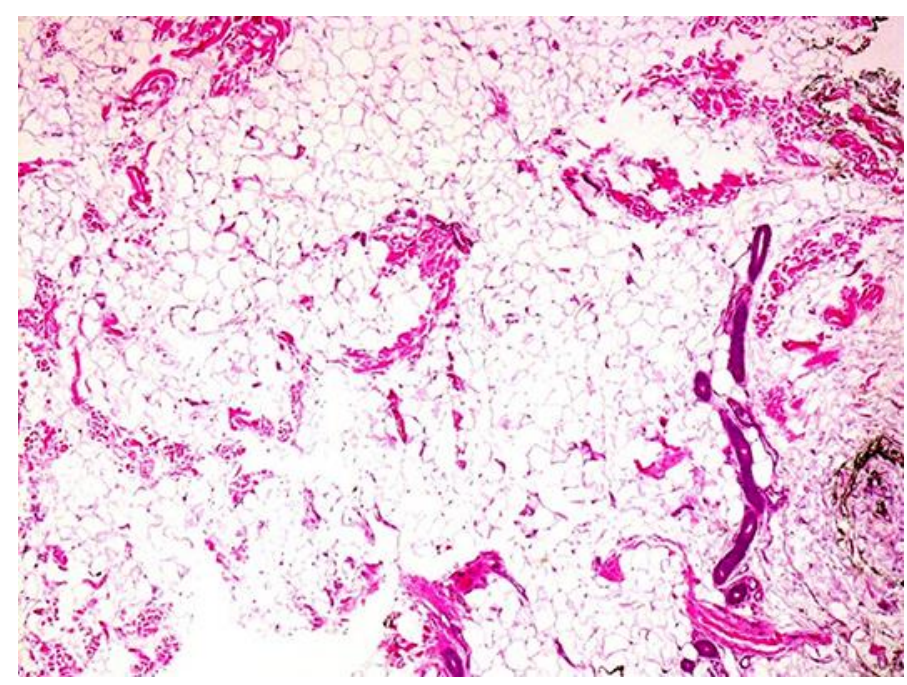

Fig. 1. Low-power microscopy showing skin with underlying subcutaneous fatty nodule. 


\section{Case Reports in Dermatology}

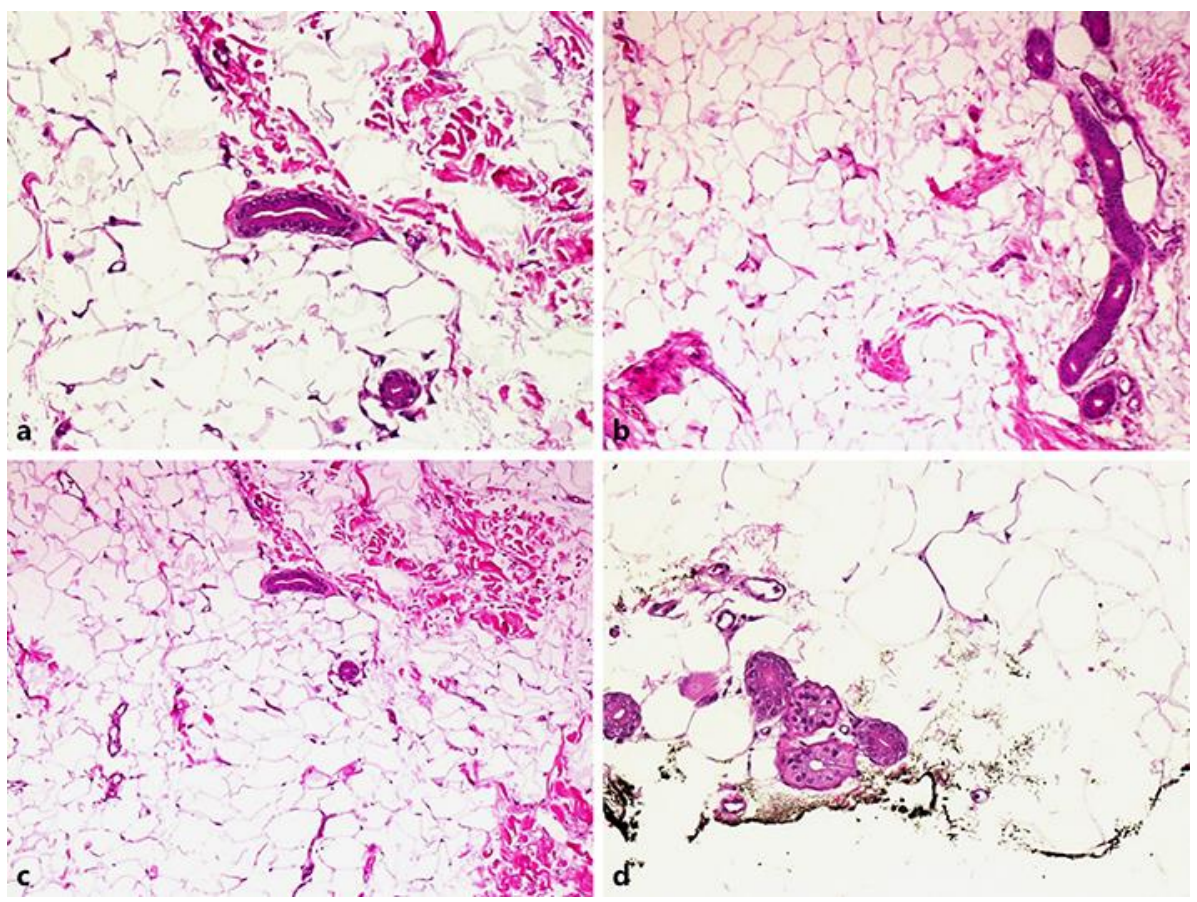

Fig. 2. a-d High-power microscopy of fatty nodule showing lobules of mature adipose tissue with scattered eccrine ducts present in between.

Table 1. Demographics and clinicopathological features of 11 cases of adenolipoma

\begin{tabular}{rlllrl}
\hline No. & Age, years & Sex, F/M & Site & Size, cm & Encapsulation \\
\hline 1 & 44 & F & Left thigh & $3 \times 1.5 \times 1$ & Present \\
2 & 41 & M & Right gluteal region & $3 \times 1.4 \times 0.7$ & Absent \\
3 & 45 & M & Right gluteal region & $5.8 \times 4.5 \times 1.8$ & Absent \\
4 & 50 & M & Right thigh & $3 \times 1.8 \times 1.5$ & Absent \\
5 & 44 & M & Left thigh & $1.6 \times 1.5 \times 1.2$ & Absent \\
6 & 53 & F & Left trunk & $2.8 \times 1.7 \times 1.1$ & Absent \\
7 & 18 & F & Left thigh & $1.8 \times 1.3 \times 0.9$ & Absent \\
8 & 44 & F & Right thigh & $2.7 \times 3 \times 1.5$ & Absent \\
9 & 79 & F & Left chest & $2 \times 1.3 \times 1$ & Absent \\
10 & 49 & M & Right lower leg & $1.5 \times 1 \times 0.3$ & Absent \\
11 & 47 & F & Left shoulder & $2.5 \times 2.5 \times 0.8$ & Absent \\
\hline
\end{tabular}

